\title{
Canulación venosa femoral distal mediante asistencia ecográfica dinámica
}

\section{Dynamic ultrasound assistance for distal femoral vein cannulation}

\author{
E. Hijosa, M. Batllori, N. Zaballos, M. Castañeda
}

\section{Sr. Director:}

La canulación venosa central es un procedimiento habitual en el ámbito hospitalario. En anestesiología la indicación para un acceso venoso central suele establecerse en el contexto perioperatorio o en el del paciente crítico y obedece fundamentalmente a la necesidad de determinadas medidas diagnósticas y/o terapéuticas ${ }^{1}$. En ocasiones también puede venir motivada por la imposibilidad para obtener un acceso venoso periférico.

El acceso venoso central a través de la vena femoral común presenta una localización relativamente segura y accesible, con la ventaja de que la anatomía del sistema venoso profundo en el triángulo femoral es consistente ${ }^{1}$. El acceso femoral evita los riesgos de hemotórax y neumotórax que presentan los accesos venosos yugular y subclavio, y se realiza a través de un punto de punción accesible a la compresión, lo cual hace de este acceso una alternativa importante en pacientes con coagulopatía y/o insuficiencia respiratoria graves. Pese a ello, es uno de los accesos venosos menos utilizados en anestesiología.
Esta escasa utilización puede explicarse en parte porque, en el ámbito del quirófano, la región femoral suele ser menos accesible que otras, como las correspondientes a los accesos yugular o subclavio. Pese a que en el triángulo femoral la vena suele localizarse medial a la arteria, no es infrecuente que exista cierto grado de solapamiento entre ambas que predisponga a una punción accidental de la arteria femoral que puede dar lugar a complicaciones de gravedad variable (sangrado, pseudoaneurisma arterial, fístula arteriovenosa). Además, tradicionalmente se ha asociado al acceso femoral una incidencia de complicaciones infecciosas y trombóticas mayor que con otros accesos venosos centra$l^{2}{ }^{2}$, lo cual suele favorecer la elección de otras alternativas.

Como consecuencia de las consideraciones expuestas, la experiencia y destreza de los anestesiólogos con la canulación femoral son considerablemente menores que con otros accesos más frecuentes. No obstante, existen situaciones en las que el acceso femoral puede ser el último recurso. La asistencia ecográfica puede facilitar el
Servicio de Anestesiología, Reanimación y Tratamiento del Dolor. Complejo Hospitalario de Navarra. Pamplona

Recepción: 15 de junio de 2012

Aceptación provisional: 3 de septiembre de 2012

Aceptación definitiva: 21 de septiembre de 2012

\section{Correspondencia:}

Esther Hijosa Basarte

Servicio de Anestesiología, Reanimación y

Tratamiento del Dolor

Complejo Hospitalario de Navarra. Irunlarrea, 3

31008 Pamplona

E-mail: niruni@gmail.com 
acceso vascular femoral en determinadas circunstancias, como en el caso que a continuación presentamos, a la vez que puede introducir modificaciones en el abordaje de punción venosa femoral ${ }^{3,4}$.

Paciente de 84 años, diagnosticado previamente de hipertensión arterial, diabetes tipo 2, obesidad importante, insuficiencia mitral moderada, fibrilación auricular crónica y asma bronquial. Fue trasladado al servicio de Urgencias de nuestro centro tras sufrir un politraumatismo por caída accidental desde un tramo de escaleras. Tras su ingreso se le diagnosticaron dos fracturas vertebrales cervicales: una en en el arco anterior $\mathrm{C} 1$ y otra en la base de la odontoides de $\mathrm{C} 2$ (fractura tipo II). Al tratarse de una fractura cervical estable, se decidió llevar a cabo inicialmente tratamiento ortopédico de la misma mediante inmovilización con collarín cervical. Asimismo se le diagnosticaron fractura supracondílea de húmero derecho, fractura de tercio proximal de húmero izquierdo y fractura metafisaria distal de radio izquierdo. Se indicó tratamiento ortopédico de las fracturas de extremidad superior izquierda, y tratamiento quirúrgico diferido de la fractura de extremidad superior derecha. En el momento del ingreso el paciente presentaba signos y síntomas de insuficiencia cardiaca (marcado edema en extremidades inferiores, disnea, ortopnea) y se encontraba anticoagulado con enoxaparina subcutánea. Ante la imposibilidad de obtener acceso venoso periférico en Urgencias, se estableció la indicación para acceso venoso central. La inmovilización ortopédica impedía las canulaciones yugular y de acceso periférico, y dadas la situación clínica del paciente y la anticoagulación establecida se desestimó la canulación subclavia. Se decidió realizar una canulación venosa femoral.

Se llevó a cabo una exploración ecográfica inicial del triángulo femoral, utilizando un transductor lineal de 13-6 MHz (HFL 38x; Sonosite, Bothell WA, EEUU). Se identificó una bifurcación "alta" de la arteria femoral común, observándose sus ramas superficial y profunda inmediatamente bajo el ligamento inguinal. La vena femoral se visualizó a
$3,5 \mathrm{~cm}$ de profundidad como un vaso compresible con flujo continuo detectado en el estudio Doppler-color. Se encontraba justo por debajo de la arteria femoral superficial, evidenciándose un solapamiento casi completo entre ambas (Fig. 1) que no pudo corregirse de manera significativa ni llevando a cabo una maniobra de abducción pasiva de la extremidad inferior ni colocando al paciente en anti-Trendelemburg. Se decidió continuar la exploración de la vena femoral siguiendo su trayecto en sentido caudal. La posición relativa de la vena respecto a la arteria fue haciéndose progresivamente más medial, hasta un punto localizado a unos $10 \mathrm{~cm}$ del triángulo femoral en el que dejó de observarse solapamiento entre ambas (Fig. 1), escogiéndose este punto para la venopunción. Ésta se llevó a cabo utilizando una técnica estéril (antisepsia cutánea, funda estéril para el transductor de ultrasonidos, bata y guantes estériles, gorro y mascarilla). Tras explorar y localizar nuevamente el punto de punción escogido, se obtuvo una imagen transversal ("en eje corto") de la vena femoral y se procedió a la punción con una aguja $18 \mathrm{G}$, mediante un abordaje "fuera de plano" con asistencia ecográfica dinámica "en tiempo real", realizado por dos exploradores. Una vez visualizada la punta de la aguja en el interior de la vena se confirmó esta situación tras obtener reflujo sanguíneo venoso a la aspiración. Se continuó la canulación empleando la técnica de Seldinger, confirmando la presencia intravascular de la guía metálica y avanzando con éxito a su través un catéter bilumen (Set Catéter venoso central 7 Fr x 20 cm, BFT (16 Ga, $16 \mathrm{Ga}$ ); Arrow, EEUU).

La canulación femoral guiada por ecografía permite llevar a cabo una evaluación más precisa de la anatomía de cada paciente, lo cual puede aumentar la proporción de punciones exitosas y reducir la incidencia de complicaciones con respecto a la técnica guiada mediante referencias anatómicas $^{5,6}$. La incorporación de la guía ecográfica ha permitido proponer un acceso femoral más distal ${ }^{2}$, que puede permitir llevar a cabo la punción en un punto más alejado de regiones potencialmente contaminables como la inguinal y perianal. 


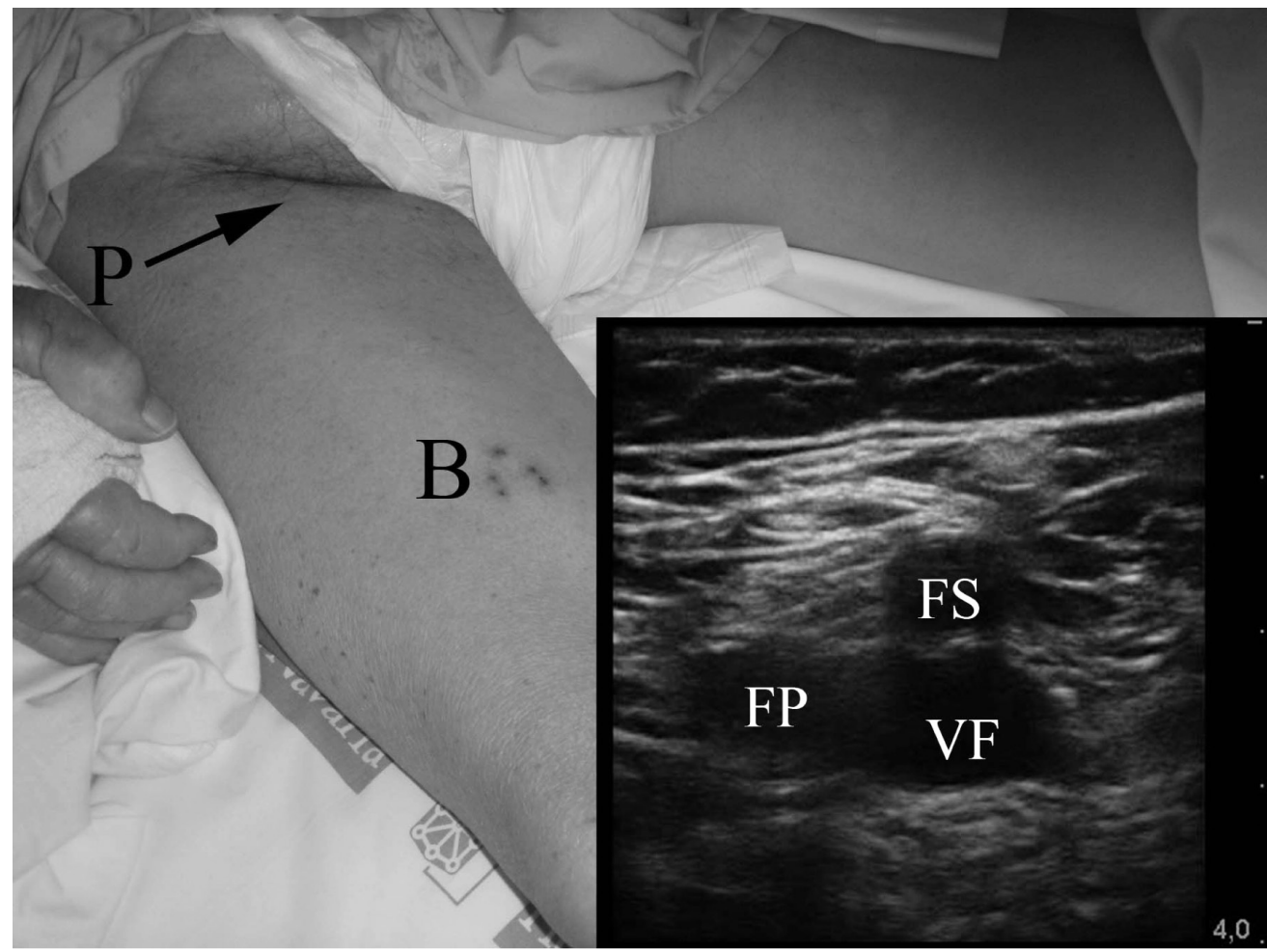

Figura 1. Exploración ecográfica vascular del triángulo femoral. La bifurcación "alta” de la arteria femoral común y la disposición de sus ramas imposibilitan la punción ecoguiada en eje corto con la aguja "fuera de plano". P punto de punción teórico, B punto de punción final, FS arteria femoral superficial, FP arteria femoral profunda, VF vena femoral.

Las últimas recomendaciones abogan por utilizar la ecografía previamente a la canulación femoral, para verificar su situación anatómica con respecto a la arteria, su permeabilidad y la facilidad de punción prevista. No existe evidencia suficiente para recomendar la asistencia dinámica respecto a la estática, si bien creemos que puede ser recomendable considerar su utilización en casos de solapamiento acusado cuando no se identifica otro punto de punción más favorable.

\section{BIBLIOGRAFÍA}

1. Beddy P, Geoghegan T, Ramesh N, Buckley O, O'Brien J, Colville J, et al. Valsalva and gravitational variability of the internal jugular vein and common femoral vein: Ultrasound assessment. Eur J Radiol 2006; 58: 307-309.
2. Wilson E, HiLlier J, FARrimond J. Ultrasound guided 'low approach' femoral vein cannulation. Anaesthesia 2004; 59: 719-729.

3. Adachi Y, Itagaki T, SuZUKI K, Uchisaki S, Kimura K, Oвата Y et al. Multiple difficulties for central venous access required the distal femoral vein catheterization: a case report. Masui 2009; 58: 913-916.

4. Sato S, Ueno E, Toyooka H. Central venous access via the distal femoral vein using ultrasound guidance. Anesthesiology 1998; 88: 838-839.

5. Prabhu MV, Juneja D, Gopal PB, Sathyanarayanan M, Subhramanyam S, Gandhe $S$, et al. Ultrasoundguide femoral dialysis access placement: a single-center randomized trial. Clin J Am Soc Neprhol 2010; 5: 235-239.

6. FARRELl J, GeLlens M. Ultrasound guided cannulation versus the landmark-guided technique for acute haemodialysis access. Nephrol Dial Transplant 1997; 12: 1234-1237. 